Crime, Histoire \& Sociétés / Crime, History \& Societies

Vol. 21, n² | 2017

L'histoire de la criminalité et de la justice pénale : propositions de recherche pour le $21^{\mathrm{e}}$ siècle

\title{
Recours à la force et demandes de justice en situation (post-)coloniale
}

\section{Emmanuel Blanchard}

\section{(2) OpenEdition \\ Journals}

Édition électronique

URL : http://journals.openedition.org/chs/1808

DOI : $10.4000 /$ chs. 1808

ISSN : 1663-4837

Éditeur

Librairie Droz

Édition imprimée

Date de publication : 31 décembre 2017

Pagination : 87-102

ISSN : 1422-0857

Référence électronique

Emmanuel Blanchard, "Recours à la force et demandes de justice en situation (post-)coloniale», Crime, Histoire \& Sociétés / Crime, History \& Societies [En ligne], Vol. 21, n² | 2017, mis en ligne le 19 juillet 2020, consulté le 15 janvier 2021. URL : http://journals.openedition.org/chs/1808 ; DOI : https:// doi.org/10.4000/chs. 1808 


\title{
Recours à la force et demandes de justice en situation (post-)coloniale
}

\author{
Emmanuel Blanchard
}

«Le problème du $\mathrm{XX}^{\mathrm{e}}$ siècle est le problème de la ligne de partage des couleurs.»

W.E.B. Du Bois, Les âmes du peuple noir (1903)

«Le monde colonisé est un monde coupé en deux.

La ligne de partage, la frontière en est indiquée par les casernes et les postes de police.»

Frantz Fanon, Les damnés de la terre (1961)

Depuis une vingtaine d'années, les études postcoloniales et les enquêtes historiques consacrées aux situations coloniales ont considérablement irrigué les recherches en criminologie et en justice criminelle, jusqu'à nourrir le récent essai A Colony in a Nation venu allonger la liste des best-sellers consacrés à la question du préjudice racial et de l'État pénal aux États-Unis ${ }^{1}$. La généalogie des violences des forces de l'ordre et de l'emprise punitive du gouvernement des États-Unis remonterait ainsi à un premier mouvement de «décolonisation» (la guerre et la déclaration d'indépendance des États-Unis) fondé sur l'affirmation de libertés ancrées dans l'exclusion raciale des Noirs et des Native Americans, autrement dit sur la formation d'une nouvelle colonie aux côtés de la jeune nation indépendante. Le détour par la situation coloniale pour décrire la situation de certaines populations étatsuniennes frappe les imaginaires contemporains mais est loin d'être inédit: à la fin des années 1960, la dénonciation du «colonialisme interne» faisait partie intégrante du répertoire rhétorique du Black power².

Surtout, au-delà des succès de librairie et des moments de mobilisation contre les violences policières, la mise en lumière des héritages de l'esclavagisme et de la ségrégation raciale est désormais classique dans les sciences sociales étatsuniennes. Elle permet notamment de rendre compte de la compartimentation raciale de la pacification du maintien de l'ordre et de la cardinalité de la rétribution dans les dispositifs de justice étatsuniens ${ }^{3}$.

Hayes (2017).

2 Blauner (1969); Gutiérrez (2004).

3 Garland (2010); Tonry (2011). 


\section{AUX ORIGINES DE LA CRIMINOLOGIE CRITIQUE}

Les analyses princeps de ce qui est devenu un véritable sous-champ de la criminologie étatsuniennes et des black studies datent de plus d'un siècle et se trouvent notamment dans l'œuvre de W.E.B. Du Bois dont la contribution à la naissance de la sociologie aux États-Unis a récemment été réévaluée 4 . Sans que les comparaisons et les emprunts ne soient toujours explicités, l'immense majorité des travaux récents sur la justice criminelle et les forces de l'ordre dans les empires européens ${ }^{5}$ a d'ailleurs retrouvé les premiers résultats de la criminologie critique proposée par Du Bois 6 .

Ces derniers sont généralement occultés par la multiplicité des engagements et des contributions de l'auteur des Âmes du peuples noir (1903) qui fut également l'un des premiers organisateurs de la défense de la cause des Afro-Américains ${ }^{7}$, un précurseur du panafricanisme et un militant engagé dans des échanges avec les principaux leaders anti-coloniaux de l'après Première Guerre mondiale ${ }^{8}$. Les liens entre criminologie et (anti-)colonialisme ne sont cependant pas réductibles à quelques personnalités emblématiques telles Du Bois mais sont profondément intriqués dans l'histoire croisée de ces projets scientifiques et politiques. Si l'on résume à très grands traits l'histoire des savoirs sur le crime et l'émergence d'une science criminologique, la criminologie critique s'est construite en opposition à une «école positiviste» longtemps dominante ${ }^{9}$. Cette dernière s'appuyait notamment sur des analyses «primitivistes» et «évolutionnistes» profondément ancrées dans une anthropologie physique qui fut un des ressorts de la légitimation des entreprises coloniales aux XIX ${ }^{\mathrm{e}}$ et $\mathrm{XX}^{\mathrm{e}}$ siècles $^{10}$.

À la même époque, certains des adversaires - notamment regroupés autour d'Émile Durkheim - de la criminologie telle que conceptualisée et mise en œuvre par Lombroso, s'attelaient à déconstruire le concept de «race» si central pour les empires coloniaux en voie de (re-)constitution au cours du long XIX ${ }^{\mathrm{e}}$ siècle $^{11}$. Ces affrontements théoriques qui contribuèrent à l'émergence d'une discipline sociologique affranchie de la raciologie n'eurent certes pas d'effets à court terme sur les dispositifs de gouvernement mis en œuvre dans les possessions ultra-marines - le «moment 1900» fut même un des apogées

Morris (2015).

5 Ces travaux peuvent relever d'orientations théoriques et méthodologiques très diverses mais ils ont en commun de s'être développés à partir de l'affaiblissement du paradigme libéral et modernisateur. Autrement dit, après que la critique de l'exportation de l'État de droit et de la professionnalisation des appareils de gouvernement se soit progressivement diffusée, à compter des années 1950, dans l'historiographie des empires européens. Voir notamment Kolsky (2010).

6 Gabbidon (2001).

7 Du Bois fut notamment l'un des fondateurs de la National Association for the Advancement of Colored People (NAACP) en 1909.

8 Sundquist (1996).

$9 \quad$ Becker et Wetzell (2006); Gabbidon (2007).

10 Blanckaert (2009). Les relations entre les tenants de l'anthropologie raciale et ceux de l'expansion européenne étaient cependant complexes. Ainsi, Paul Broca était critique de la «subordination des races et du colonialisme défendu par nombre des membres de la Société d'anthropologie de Paris. Wartelle (2004); Conklin (2013).

11 Mucchielli (1997). 
des violences coloniales, avec notamment les massacres génocidaires au Sud-Ouest africain allemand ${ }^{12}$.

Ils contribuèrent cependant, à long terme, à délégitimer des entreprises coloniales qui avaient été intimement adossées à la constitution de nouveaux savoirs scientifiques et à la formation de nouveaux domaines académiques ${ }^{13}$.

\section{COLONIES PÉNALES}

Les territoires coloniaux furent également les terrains d'expérimentation des politiques les plus répressives, notamment pour les déportés et relégués européens, transportés depuis les métropoles: ils étaient paradoxalement appelés à une «mort sociale» mais aussi à alimenter une colonisation pénale présentée comme un remède aux angoisses démographiques des tenants de la conquête et de l'appropriation territoriale ${ }^{14}$.

Les empires coloniaux furent également le lieu d'expérimentation de quelquesunes des techniques des sciences criminelles emblématiques de la modernité policière, telles la dactyloscopie dont certains des fondements furent inaugurés au Bengale ${ }^{15}$. Surtout, les habitants autochtones de ces territoires, quand ils échappèrent aux entreprises d'extermination liées au settler colonialism, furent soumis à une logique du «surplus pénal» et à un «réseau coercitif» particulièrement serré16.

Ce poids de «l'État pénal» ou du «pouvoir despotique» se mesurait parfois en termes absolus - au travers du nombre de policiers, militaires et autres «corps habillés»- notamment dans certaines colonies pénitentiaires britanniques ${ }^{17}$. Les territoires colonisés furent cependant, dans l'immense majorité des cas, loin d'être des États policiers, du moins si l'on s'en tient à la mesure des effectifs de l'appareil pénal ou à l'estimation des densités policières.

Il en est de même lorsque l'on examine la capacité de leurs services de renseignement à enregistrer les populations et à fournir des informations rendant compte des évolutions politiques et sociales, notamment dans les régions rurales ${ }^{18}$. «L'anxiété épistémologique» de colons et de gouvernants conscients qu'ils ne maitrisaient ni les clivages, ni les ressorts des formations sociales qu'ils étaient supposés administrer fut ainsi une des caractéristiques profondes des situations coloniales ${ }^{19}$.

Le poids de «l'État pénal» aux colonies était fondamentalement lié au manque d'outils cognitifs, et pas seulement aux faibles moyens matériels des autres secteurs d'intervention étatiques. Ces derniers ne prirent une relative ampleur qu' après la Seconde Guerre mondiale, quand le paradigme du «développement» remplaça celui de la «mission

\footnotetext{
Eckl (2008).

Bourdieu (1976); Singaravélou (2011).

Anderson (2016); Sanchez (2013).

Cole (2001).

6 Wolfe (1999); Elkins et Pedersen (2005); Brown (2002); Sherman (2009).

17 Mann (1984); Debos et Glasman (2012); Petrow (2000, 2011).

18 Bayly (1993); Thomas (2008)

19 Stoler (2009).
} 
de civilisation», voire de la «supériorité raciale $»^{20}$. Des effectifs souvent faibles en valeurs absolus ont souvent retenu l'attention des spécialistes des administrations coloniales. Le primat des appareils répressifs fut cependant incontestable en regard du poids relatif de «l'État infrastructurel» et des effectifs des autres administrations coloniales $^{21}$. Au-delà des questionnements sur la formation et le présent de l'État dans les anciennes colonies européennes, cette configuration étatique nourrit les comparaisons contemporaines avec un certain nombre de territoires où le retrait de l'État social s'est accompagné d'une montée de l'emprise policière et de l'État pénal ${ }^{22}$.

L'État colonial n'était pas forcément centralisé: il s'appuyait généralement sur des autorités ou des lignes de commandements vernaculaires, et il s'est bien souvent réduit à ses dimensions dites de souverainetée ${ }^{23}$. Ces dernières étaient cristallisées dans l'administration d'une violence sans limite, considérée comme la seule réponse aux tentatives de soulèvement ou de contestation. Elle s'appuyait notamment sur la mise en œuvre d'un droit de punir aux périmètres élargis, incarné particulièrement dans les Criminal tribes act de l'empire britannique ou le régime de l'indigénat dans l'empire français ${ }^{24}$.

Ce primat de la force comme instrument de gouvernement - même dans l'empire britannique où le modèle du constable fut exporté, le policing by consent ne fit jamais véritablement figure d'horizon du maintien de l'ordre - se perpétua, voire se renforça, jusqu'à la fin des occupations coloniales: les atrocités perpétrées, au travers notamment des regroupements de population, en Indonésie, au Kenya ou en Algérie illustrent que les autorités coloniales, qu'elles soient hollandaises, britanniques ou françaises, n'avaient pas véritablement d'autres projets et modes de légitimation à mettre en œuvre ${ }^{25}$.

L'après Seconde Guerre mondiale, période pendant laquelle les puissances impériales commencèrent à développer véritablement leurs appareils de santé ou de scolarisation - parfois sous le contrôle et avec la contribution des militaires -, fut aussi le moment où les effectifs de police ou de gendarmerie augmentèrent le plus rapidement en lien avec l'accroissement du nombre des recrues autochtones. Ces transformations de l'époque des décolonisations constituèrent la base coercitive de nombreux États dits post-coloniaux et inscrivirent ces systèmes de contraintes au cœur des «durabilités» et «legs coloniaux $»^{26}$.

\section{DE LA POST-COLONIE}

Les «effets de retour» de cette punitivité spectaculaire et des techniques policières appliquées outre-mer furent nombreux: le fichage dactyloscopique, l'usage des armes contre les manifestants, ou l'histoire de la peine de mort et de son abolition tardive, en particulier en France - en 1981, après qu'elle ait été appliquée

\footnotetext{
$20 \quad$ Hodge et al. (2014); Osborne (2014)

21 Mann (1984); Glasman (2015).

22 Berman et Lonsdale (1992); Bayart (1996); Wacquant (2009); Donzelot (2013); Goffman (2014).

23 Steinmetz (2008).

24 Fourcade (1994); Major (1999); Mann (2009); Thénault (2012).

25 Arnold (1986); Branche (2001); Locher-Scholten (2002); Anderson (2005).

26 Anderson et Killingray (2012); Houte et Luc (2016); Bayart et Bertrand (2006); Stoler (2016).
} 
plus que jamais, depuis son «abolition» pour «motifs politiques» en 1848, pendant la guerre d'indépendance algérienne ${ }^{27}$ - ont emprunté des chemins qui passèrent par les colonies ${ }^{28}$. L'avènement des «indépendances» n'a pas fait entrer les métropoles des anciennes formations impériales dans une ère radicalement nouvelle en matière de maintien de l'ordre et de justice criminelle.

Ainsi, les contrôles d'identité et les rafles de «Français musulmans » qui s'étaient multipliés dès la fin des années 1940 dans les régions d'importante immigration algérienne, se sont-ils reportés, à partir du milieu des années 1960, sur les jeunes hommes «arabes» vivants dans des «banlieues». Ces dernières furent peu à peu construites en problème public générateur de paniques morales relatives à la criminalité et à la délinquance juvénile, de plus en plus appréhendées selon des grilles de lecture racialisées ${ }^{29}$.

En ce domaine, de nombreux héritages coloniaux peuvent d'ailleurs être mis au jour par l'enquête historique: ainsi, dans le cas français, la carte nationale d'identité a été instituée comme instrument de contrôle des déplacements en 1955, à la faveur du conflit algérien ${ }^{30}$. Avant ce même conflit, les contrôles d'identité et les rafles touchaient déjà certains groupes de «citoyens diminués» (prostituées, homosexuels, vagabonds, tsiganes, etc.). Ils ne constituaient cependant pas une technique routinière des «gardiens de la paix» et ne s'appliquaient pas à des fractions aussi larges des habitants des quartiers pauvres ${ }^{31}$. Cette cardinalité des contrôles d'identité dans la culture professionnelle des policiers français ne manque pas de susciter l'étonnement de leurs homologues de pays voisins, aux passés coloniaux contrastés, mais qui utilisent rarement ces «cérémonies de dégradation» afin d'entrer en contact avec leurs administrés ${ }^{32}$. Il est ainsi indéniable que le «style policier» français, caractérisé par sa rugosité et sa distance dans les rapports avec la population, a profondément été marqué par les guerres de décolonisation même s'il s'ancre aussi dans la longue durée d'une police avant tout pensée comme étant au service du souverain puis de l'Etat ${ }^{33}$.

Passant outre ces historicités mêlées, certains auteurs n'hésitent cependant pas à tracer des généalogies plus globales entre la condition des colonisés et celle des habitants des quartiers de relégation des villes mondialisées, dont une partie - mais une partie seulement - sont des descendants des premiers ${ }^{34}$. Dans des contextes où les objectivations historiques et statistiques sont des exercices particulièrement délicats, les continuités post-coloniales semblent plus souvent affirmées théoriquement que démontrées empiriquement, à tel point que l'on peut s'interroger sur leur plus-value heuristique: la mise en exergue de la (post-)colonialité ne vient-elle pas redoubler la

27 Les environ 220 combattants de l'Armée de libération nationale (ALN) qui furent exécutés entre 1956 et 1962, l'ont été après des condamnations de «droit commun » prononcées par des tribunaux, tant civils que militaires, en métropole et en Algérie (Thénault, 2001; Malye et Stora, 2010).

28 Foucault (1997).

29 Mohammed et Mucchielli (2007).

30 Piazza (2004).

31 Blanchard (2008).

32 Blanchard (2014); Gauthier et Lucas (2011); De Maillard et al. (2016).

33 Berlière et Lévy (2011).

34 Sassen (2015). 
démonstration de la racialisation des pratiques de maintien de l'ordre ${ }^{35}$, dans un effort de mise au jour non pas simplement de constats mais de causalités ${ }^{36}$ ? Or, selon les pays, quelles que soient les formes que prend la colour bar et, plus spécifiquement, la domination policière, elle est le fruit d'une combinatoire de multiples critères de hiérarchisation des populations, dont le passé colonial - qui peut être partagé par des pays n'ayant pas constitué de véritables empires - n'est qu'une des modalités ${ }^{37}$.

Il reste que, selon des modalités et des trajectoires différentes, en fonction des régions et des époques, les questions pénales et coloniales ont été étroitement intriquées et ont participé d'une même configuration socio-raciale. La colonialité du pouvoir ne fut pas tant circonscrite par des frontières géographiques que par des dispositifs pénaux (les droits à circuler, l'emprise carcérale, les modalités des peines, etc.), des processus de (non-) reconnaissance ou la prégnance de certaines représentations qui lui donnèrent des formes de consistance jusqu'au cœur des métropoles impériales ${ }^{38}$. L'importance du passé esclavagiste et de l'expansion coloniale dans la genèse des sociétés (post-)industrielles contemporaines demeure d'ailleurs une des caractéristiques (dont les inégalités socio-raciales donnent un proxy statistique) permettant de rendre compte des différences d'intensité de la punitivité et de l'emprise pénale des États actuels ${ }^{39}$.

On observe d'ailleurs une forme de consensus scientifique sur cette question, les enjeux de recherches contemporains étant surtout liés aux méthodologies d'enquête permettant de capter la colonialité et son articulation avec d'autres formes de domination et de régulation sociale. Surtout, bien plus qu'un enjeu scientifique, la prise en compte du préjudice de race vécu par une partie des clientèles policières subissant, sous des formes actualisées, les pratiques de «surplus pénal» infligées aux indigènes à la période coloniale, demeure un enjeu politique et militant. De la connaissance à la reconnaissance, du constat scientifique à la prise en compte dans les politiques publiques, le chemin est long et implique nécessairement que de nouveaux travaux et des prises de position réitérées viennent rappeler que le "passé n'est pas mort et enterré $»^{40}$.

Dans le champ scientifique, même si le style, les insistances et les hiérarchies énonciatives peuvent varier, il y a au moins là une forme de consensus, seulement fragilisé par la difficulté d'exprimer à nouveaux frais les savoirs cumulés.

35 Sur le contexte étatsunien, la littérature est particulièrement abondante et, parmi les travaux récents, on peut notamment faire référence à Hagan (2010) et Hinton (2016). Dans le cas français, les travaux se multiplient tant sur les contrôles d'identité (Jobard et Lévy, 2009), le sentencing (Jobard et Nevanen, 2007) que la prison (Fassin, 2015). Ils restent cependant comparativement peu nombreux.

36 Mbembe (2001); Stoler (2016).

37 Särkkä (2015).

38 Quijano (2000); Epple et Lindner (2011); Blanchard (2011).

39 Tonry $(2007,2009)$.

40 Cette citation de William Faulkner («The past isn’t dead and buried. In fact, it isn’t even past» Requiem for a Nun, 1951), très souvent mobilisée, a notamment été utilisée par Barack Obama, alors candidat aux élections présidentielles, dans un de ses rares discours consacrés à la question raciale (mars 2008). Elle est souvent reprise par des militants dénonçant les violences policières et rappelant que les Black lives matter. 


\section{RECOURS EN JUSTICE ET PLURALISME JURIDIQUE}

La «nouvelle histoire impériale» a ainsi emprunté des voies de traverse contournant la focalisation sur les seuls mécanismes punitifs. Il n'y a en effet plus guère de chercheurs pour contester que la politique pénale aux colonies s'est organisée selon un «principe de différence» largement racialisée ${ }^{41}$. Mais celui-ci était loin de s'exprimer seulement dans les termes de la violence et des peines afflictives. Les situations coloniales ont avant tout été caractérisées par un pluralisme juridique et juridictionnel qui permet de nuancer le grand récit de la rationalisation judiciaire qui aurait marqué les démocraties occidentales nées de la sortie progressive de «l'Ancien Régime».

L'État colonial s'est ainsi caractérisé par la prolongation aux XIX ${ }^{e}$ et $\mathrm{XX}^{\mathrm{e}}$ siècles de dispositifs de gouvernement (la multiplicité des textes et des juridictions, les corvées, le travail forcé, les bagnes, le contrôle des circulations) supposément caractéristiques de la période précédente ${ }^{42}$. Les puissances impériales ont également largement contribué à une «invention de la tradition», en matière de droit (pénal mais aussi civil) et de justice, dans les principales régions colonisées ${ }^{43}$. La multiplicité des acteurs de la justice, des sources du droit, le pluralisme juridique et les chevauchements juridictionnels n'étaient pas seulement un objet d'étude pour les spécialistes d'un droit colonial dont une large part était déjà perçue par certains de ces mêmes spécialistes comme un véritable «monstre juridique ${ }^{44}$. Le «flou du droit» permettait certes la réaffirmation d'une souveraineté sans contre-pouvoir mais donnait aussi de véritables marges de manœuvre aux subalternes ${ }^{45}$.

Au-delà des suppliques et autres pétitions adressées en nombre aux autorités coloniales, l'on vit se multiplier, à partir du début du $\mathrm{XX}^{\mathrm{e}}$ siècle, les saisines des tribunaux, une même affaire étant bien souvent portée devant plusieurs juridictions. Ces mouvements de contestation juridique et ces multiples tentatives de faire valoir ses droits - en particulier en matière civile - ne doivent pas seulement être interprétés comme des signaux de capacités d'action trop peu prises en compte par les travaux focalisés sur les violences coloniales ${ }^{46}$. Ces saisines étaient aussi la conséquence de formes multiples de dépossessions et d'entraves à l'expression publique: c'est notamment parce qu'ils avaient été expropriés de leurs terres, ou fragilisés par les réformes foncières dans leurs capacités d'attestation de leurs possessions, que les colonisés devaient si souvent ester en justice pour faire reconnaître ou sécuriser leurs droits de propriétét ${ }^{47}$.

C'est également au nom du principe de «civilisation» que les régimes matrimoniaux avaient été l'objet de réformes: les demandes de divorce ou les recours pour abandon de famille étaient donc nombreux à aboutir devant la justice coloniale, sans que cette dernière réussisse cependant à saisir, ni même enregistrer l'ensemble des arrangements matrimoniaux. Plus généralement, l'accès à l'arène politique étant fermé, ou à peine

\footnotetext{
Chatterjee (1994); Kolsky (2005).

42 Fremigacci (2014).

43 Hobsbawm et Ranger (1983); Benton (2002).

44 Blévis (2005); Renucci (2011); Benton (2009).

45 Delmas-Marty (2004).

46 Roberts (2005); Rodet (2009); Burrill (2015).

47 Weaver (2003); Guignard (2013).
} 
entrouvert (avec un droit de vote accordé aux seuls «évolués» ou des syndicats longtemps interdits) et très coûteux (journaux censurés, manifestations réprimées, etc.), l'arène et les professions judiciaires furent centrales dans la contestation coloniale ${ }^{48}$.

Dans les anciennes métropoles impériales, ces barrières contemporaines sont rarement juridiques - le critère de nationalité continue cependant d'opérer notamment en matière de vote et d'emplois publics - mais des formes de «cens caché» continuent de peser sur celles et ceux qui se présentent comme les descendants de cette immigration coloniale. À cet égard, la judiciarisation de leur action politique, en particulier quand elles visent les violences policières, peut certes être interprétée comme un élargissement de leur répertoire d'action. Mais elle signale également une limite, voire une impasse, si elle est la seule voie permettant de surmonter des mécanismes pérennes d'entraves à d'autres formes de prises de paroles et d'actions politiques ${ }^{49}$.

\section{DEMANDES DE POLICE}

Paradoxalement, pour les subalternes, la situation coloniale se caractérisait autant par une absence de police que par «l'excès pénal» généralement mis au jour par l'histoire impériale des peines. De fait, les «Européens »- autrement dit, les «blancs » puisque les démarcations raciales étaient primordiales même si elles ne se réduisaient pas à un colorisme sans nuances - bénéficiaient d'un triple privilège juridictionnel: les forces de l'ordre protégeaient avant tout les espaces de résidence des colons ainsi que les lieux de production tournés vers l'exportation des commodités coloniales; ces mêmes «blancs» échappaient généralement à toute sanction policière émanant d'un agent colonisé, ces derniers ne pouvant poursuivre que des subalternes; en revanche, en l'absence de forces de l'ordre institutionnalisées et situées à proximité, les colons bénéficiaient tous de pouvoirs de police informels leur permettant d'user «avec discernement» de la force afin de défendre leur «honorabilité » et leur autorité auprès des subalternes, en particulier sur celles et ceux travaillant dans les espaces économiques où les polices privées étaient légion ${ }^{50}$.

D'une manière générale, la police du quotidien était relativement peu développée: la défense des intérêts économiques des prépondérants coloniaux et les questions politiques étaient placées au cœur du travail des forces de l'ordre. Un certain rééquilibrage fut cependant observé pendant l'entre-deux-guerres ${ }^{51}$. Cela tenait à la fois au projet de discipliner les autochtones de plus en en plus nombreux à venir rejoindre les quartiers pauvres des villes coloniales mais également à ce que les classes moyennes locales émergentes faisaient entendre leur voix au travers d'argumentaires qui ne pouvaient être balayés sans discussion par les autorités coloniales: à la revendication de l'extension des droits individuels et salariaux s'ajoutaient de nombreuses demandes de renforcement des services (éducation, santé, sécurité...) promis à la population ${ }^{52}$. Or,

\footnotetext{
48 Gobe (2013).

49 Jobard (2006).

50 Blanchard et al. (2017); Thomas (2012); Anderson (1994); Kolsky (2010).

51 Brunet (2012).

52 Cooper (1996).
} 
à cette époque, légitimation de la présence coloniale oblige, la fiscalité ne pouvait plus se résumer à une extraction de richesses et devait avoir pour pendant des contreparties tangibles pour les gouvernés. La question de «l'insécurité» devint alors cardinale, non plus seulement au sens des dangers pesant sur la souveraineté coloniale - chaque atteinte aux colons était perçue comme mettant à mal cette dernière.

Il s'agissait également d'une traduction de la difficulté à répondre aux attentes d'une partie des subalternes en matière de protection des biens et des personnes ${ }^{53}$. L'incapacité de l'État colonial à répondre à ces demandes eut des conséquences majeures: cela contribua à son affaiblissement et le renvoya à une véritable aporie - si les ressources fiscales devaient alimenter une véritable politique de «services publics » et de redistribution, la politique coloniale devenait trop coûteuse et perdait toute légitimité dans les métropoles ${ }^{54}$. Cela conduisit également à des formes de pluralisme policier et de vigilantism qui, jusqu'à nos jours, marquent certaines de ces régions, notamment en Afrique du Sud et de l'Ouest ${ }^{55}$. Les espaces non investis par les autorités coloniales virent en effet émerger des milices, big men et autres vigilantes. Puis ces derniers répondaient à une partie des attentes de sécurité des habitants, tout en s'opposant éventuellement aux forces de l'ordre «centrales» dont l'irruption venait perturber un ordre local construit en dépit de leur absence structurelle ${ }^{56}$.

L'analyse des forces de police coloniale, «par le bas» et en particulier dans les zones rurales, a récemment contribué à renouveler l'historiographie du colonial policing, par une attention particulière à des logiques professionnelles venant infléchir les grands cadres politico-administratifs ${ }^{57}$. L'approfondissement de ces pistes de recherche devra cependant passer par une attention centrale à la question de la demande de police, loin de se résumer à la capacité à remodeler localement les dispositifs pensés au niveau central ${ }^{58}$. Intégrer pleinement la demande de police aux études sur le maintien de l'ordre permettrait de restituer toute la palette des attentes et des requêtes de populations colonisées pour lesquelles le principe de sécurité était également cardinal et s'exprima en des formes de plus en plus institutionnalisées.

En cela, l'historiographie des polices suivrait celle de la justice pour laquelle l'étude des modalités d'action des justiciables et autres requérants tentant de faire valoir leurs droits est devenue centrale ${ }^{59}$. La description de l'État colonial gagnerait ainsi en précision et pourrait faire ressortir de nouvelles complexités et fragilités. Il apparaissait en effet aux gouvernants coloniaux, certes ouverts à des formes de délégation de l'usage de la force ${ }^{60}$, qu'il n'appartenait pas aux colonisés de définir ce que devaient être les priorités de la sécurité et du maintien de l'ordre.

Incidemment, de tels travaux permettraient également de renouveler les questionnements sur les continuités coloniales: les territoires contemporains dits marqués par la résurgence de dispositifs coloniaux et la militarisation du maintien de l'ordre ne

\footnotetext{
53 Bloembergen (2012).

54 Lefeuvre (1997).

55 Fourchard $(2008,2011)$

56 Berg et Wendt (2011); Favarel-Garrigues et Gayer (2016).

57 Giulani (2015).

58 Chandavarkar (1998); Kidambi (2004).

59 Roberts (2013).

60 Emsley (2014).
} 
souffrent pas seulement d'une trop grande emprise policière mais concomitamment et paradoxalement - de la faiblesse de certaines formes de présence policière. Ainsi, quelles que soient les modalités et les configurations institutionnelles du community policing, il a rarement été mis en œuvre de manière pérenne dans les quartiers les plus pauvres et considérés comme les plus touchés par la criminalité6 ${ }^{1}$.

Quand des forces policières se sont installées au cœur de lieux de relégation - dont elles contrôlent généralement les marges sans guère y assurer une présence continue - cela a bien souvent été sous la forme d'une police militarisée particulièrement violente - l'on peut notamment penser à certaines favelas de Rio investies en force ces dernières années ${ }^{62}$. Le vocabulaire utilisé afin de qualifier ces «opérations» («occupation», «pacification») signale à lui seul les legs coloniaux à l'œuvre dans de tels cas $^{63}$. Les niveaux de violence policière atteints ces dernières années au Brésil sont de longue date parmi les plus élevés du monde et ne sont certes pas comparables à ceux observés dans les anciennes métropoles coloniales ${ }^{64}$.

Il reste que dans de nombreuses régions du monde, quel que soit leur niveau de développement, les attentes des habitants des quartiers pauvres en matière de sécurité sont peu prises en compte, quand elles ne sont pas tout simplement disqualifiées comme nourrissant le crime. Or, les enquêtes de victimation démontrent sans conteste que l'indifférence ou la défiance par rapport à la police sont particulièrement fortes dans ces territoires où elles se nourrissent à la fois de ce que la police apparaît peu protectrice, inefficace mais également peu à l'écoute de celles et ceux qui souhaiteraient faire appel à elle autrement qu'en incarnant la figure de l'informateur ${ }^{65}$. Dans les modalités de mises en contact avec les forces de l'ordre, ces habitants sont en effet plus perçus comme des sources potentielles de renseignement que comme des citoyens ayant une vision de l'ordre à protéger et à partager. En cela, ils partagent bien la condition des subalternes de la période coloniale.

La question de savoir dans quelle mesure cette méfiance et cette distance tiennent à des héritages coloniaux (en termes de représentations racialisées, d'organisations et de dispositifs policiers) trouvera des réponses dans de nouvelles enquêtes socio-historiques: elle ne peut en tout cas pas être balayée du seul fait que ces configurations des rapports police-population tiennent forcément à des généalogies multiples ancrées dans des contextes locaux.

\section{Emmanuel Blanchard} CESDIP-CNRS

UMR 8183, Guyancourt blanchard@ cesdip.fr

\footnotetext{
$61 \quad$ Skogan (2003).

62 Riccio et al. (2013); Amoroso et al. (2014).

63 O'Reilly (2017).

64 Il est particulièrement complexe de calculer des taux d'homicides perpétrés par des policiers, ce type de statistiques n'étant en général pas récolées, ou sous-évaluées, par les administrations étatiques (Fyfe, 2002; Barber et al., 2016). On peut cependant retenir comme ordre de grandeur que chaque année, en France, on compte environ une dizaine de décès en lien avec une intervention policière. Au Brésil, plus d'un millier de personnes (dont plusieurs centaines pour la seule ville de Rio avec, selon Amnesty International, une pointe à 920 en 2016) tombent chaque année sous les balles policières.
} 


\section{BIBLIOGRAPHIE}

Amoroso, M., Brum, M. et Gonçalves, R. (2014) Police, participation et accès aux droits dans des favelas de Rio de Janeiro: l'expérience des Unités de police de pacification (UPP), Revue Orda. L'Ordinaire des Amériques, 216 [En ligne] http://journals.openedition.org/ orda/1014

Anderson, C. (2016) Transnational Histories of Penal Transportation: Punishment, Labour and Governance in the British Imperial World, 1788-1939, Australian Historical Studies, 47, 3, p. 381-397.

Anderson, D. (1994) Policing the Settler State: Colonial Hegemony in Kenya, 1900-1952, in Engels, D., Marks, S. (Eds.), Contesting Colonial Hegemony. State and Society in Africa and India, London: British Academic Press/The German Historical Institute, p. 248-264.

Anderson, D. (2005) Histories of the Hanged: The Dirty War in Kenya and the End of Empire, New York: W.W. Norton.

Anderson, D. et Killingray, D. (1992) Policing and Decolonisation: Politics, Nationalism, and the Police, 1917-65, Manchester University Press.

Arnold, D. (1986) Police Power and Colonial Rule, Madras, 1859-1947, Oxford University Press.

Barber, C., Azrael D., Cohen, A. et al. (2016) Homicides by Police: Comparing Counts From the National Violent Death Reporting System, Vital Statistics, and Supplementary Homicide Reports, American Journal of Public Health, 106, 5, p. 922-927.

Bayart, J.-F. (Dir.) (1996) La greffe de l'État, Paris: Karthala.

Bayart, J.-F. et Bertrand, R. (2006) De quel « legs colonial » parle-t-on ?», Esprit, [En ligne] https://esprit.presse.fr/article/bayart-jean-francois-et-bertrand-romain/de-quel-legscolonial-parle-t-on-13808

Bayly, C. (1993) Knowing the Country: Empire and Information in India, Modern Asian Studies, 27, 1, p. 3-43.

Becker, P., Wetzell, R. (Eds.) (2006) Criminals and their Scientists: The History of Criminology in International Perspective, Cambridge: University Press.

Benton, L. (2002) Law and Colonial Cultures: Legal Regimes in World History, 1400-1900, Cambridge University Press.

Benton, L. (2009) A Search for Sovereignty: Law and Geography in European Empires, 14001900, Cambridge University Press.

Berg, M., Wendt, S. (Eds.) (2011) Globalizing Lynching History: Vigilantism and Extralegal Punishment from an International Perspective, London: Palgrave Macmillan.

Berlière, J.-M. et Lévy, R. (2011) Histoire des polices de l'Ancien Régime à nos jours, Paris: Nouveau Monde.

Berman, B. et Lonsdale, J. (1992) Unhappy Valley: Clans, Class and State in Colonial Kenya, Athens: Ohio University Press.

Blanchard, E. (2008) Encadrer des «citoyens diminués». La police des Algériens en région parisienne, 1944-1962 (thèse d'histoire, université de Dijon).

Blanchard, E. (2011) La police parisienne et les Algériens, 1944-1962, Paris: Nouveau monde. Blanchard, E. (2014) Contrôles au faciès : une cérémonie de dégradation, Plein Droit, 103, p. 15-20. 
Blanchard, E., Bloembergen, M. et Lauro A. (Eds.) (2017) Policing in colonial empires: cases, connections, boundaries (ca. 1850-1970), Bruxelles: P.I.E. Peter Lang.

Blanckaert, C. (2009) De la race à l'évolution: Paul Broca et l'anthropologie française (18501900), Paris: Harmattan.

Blauner, R. (1969) Internal colonialism and ghetto revolt, Social Problems, 16, 4, p. 393-408.

Blévis, L. (2005) Juristes et légistes au service de l'État colonial. De la mise en forme technique du statut des Algériens à sa difficile légitimation, in Israël, L., Sacriste, G. et Vauchez, A. (Eds.), Sur la portée sociale du droit, Paris: PUF-CURAPP, p. 309-324.

Bloembergen, M. (2012) Vol, meurtre et action policière dans les villages javanais. Les dynamiques locales de la sécurité aux Indes néerlandaises orientales dans les années 1930, Genèses, 86, p. 8-36.

Bourdieu, P. (1976) Les conditions sociales de la production sociologique. Sociologie coloniale et décolonisation de la sociologie, in Moniot, H. (Dir.), Le mal de voir, Paris: UGE, coll. «10/18», p. 416-427.

Branche, R. (2001) La torture et l'armée pendant la guerre d'Algérie (1954-1962), Paris: Gallimard.

Brown, M. (2002) The politics of penal excess and the echo of colonial penality, Punishment \& Society, 4, 4, p. 403-423.

Burrill, E. (2015) States of marriage: gender, justice, and rights in colonial Mali, Athens: Ohio University Press.

Chandavarkar, R. (1998) Imperial power and popular politics: class, resistance and the state in India, c. 1850-1950, Cambridge University Press.

Chatterjee, P. (1994) The nation and its fragments: Colonial and postcolonial histories, Princeton University Press.

Cole, S. (2001) Suspect Identities. A History of Fingerprintings and Criminal Identification, Cambridge Mass.: Harvard University Press.

Conklin, A. (2013) In the Museum of Man: Race, Anthropology, and Empire in France, 18501950, Ithaca: Cornell University Press.

Cooper, F. (1996) Decolonization and African Society: The Labor Question in French and British Africa: Cambridge University Press.

Debos, M. et Glasman, J. (2012) Politique des corps habillés. État, pouvoir et métiers de l'ordre en Afrique, Politique africaine, 128, p. 5-23.

Delmas-Marty, M. (2004) Le flou du droit: du Code pénal aux droits de l'homme, Paris: PUF.

De Maillard, J.; Hunold, D., Roché, S. et al. (2016) Les logiques professionnelles et politiques du contrôle. Des styles de police différents en France et en Allemagne, Revue française de science politique, 66, 2, p. 271-293.

Eckl, A. (2008) The Herero genocide of 1904: Source-critical and methodological considerations, Journal of Namibian Studies, 3, p.31-61.

Elkins, C., Pedersen, S. (2005), Settler Colonialism in the Twentieth Century: Projects, Practices, Legacies: Routledge.

Emsley, C. (2014) Policing the empire/Policing the metropole: Some thoughts on models and types, Crime, Histoire \& Sociétés/Crime, History \& Societies, 18, 2, p.5-25. 
Epple, A. et Lindner, U. (2011) Entangled Histories: Reflecting on Concepts of Coloniality and Postcoloniality, Comparativ|Zeitschrift für Globalgeschichte und vergleichende Gesellschaftsforschung, 21, p. 7-13.

Fassin, D. (2015) L'ombre du monde: une anthropologie de la condition carcérale, Paris: éd. du Seuil.

Favarel-Garrigues, G. et Gayer, L. (2016) Violer la loi pour maintenir l'ordre, Politix, 115, p. 7-33.

Foucault, M. (1977) Il faut défendre la société: cours au Collège de France, 1975-1976, Paris: Gallimard-Seuil, 1997.

Fourcade, M. (1994) Les dénommées «tribus criminelles» de l'Inde britannique: violence coloniale, violence traditionnelle, Purusārtha, 16, p. 187-211.

Fourchard L. (2008) A New Name for an Old Practice: Vigilantes in South-Western Nigeria, Africa: The Journal of the International African Institute, 78, 1, p. 535-558.

Fourchard, L. (2011) The Politics of Mobilization for Security in South African Townships, African Affairs, 110, 441, p. 607-627.

Fremigacci, J. (2014) État, économie et société coloniale à Madagascar: de la fin du XIX $X^{e}$ siècle aux années 1940, Paris: Karthala.

Fyfe, J. (2002) Too Many Missing Cases: Holes in our Knowledge about Police Use of Force, Justice Research and Policy, 4, 1-2, p. 87-102.

Gabbidon, S. (2001) W.E.B. Du Bois: Pioneering American Criminologist, Journal of Black Studies, 31, 5, p. 581-599.

Gabbidon, S. (2007) W.E.B. Du Bois on Crime and Justice: Laying the Foundations of Sociological Criminology, Ashgate Publishing.

Garland, D (2010) Peculiar Institution: America's Death Penalty in an Age of Abolition, Cambridge Mass: Belknap Press.

Gauthier, J. et Lukas, T. (2011) Warum kontrolliert die Polizei (nicht)? Unterschiede im Handlungsrepertoire deutscher und französischer Polizisten, Soziale Probleme, 22, 2, p. 174-206.

Glasman, J. (2015) Les corps habillés au Togo. Genèse coloniale des métiers de police, Paris: Karthala.

Gobe, É. (2013) Les avocats en Tunisie de la colonisation à la révolution (1883-2011): sociohistoire d'une profession politique, Paris: Karthala.

Goffman, A. (2014) On the Run: Fugitive Life in an American City, The University of Chicago Press.

Guignard, D. (2013) Les inventeurs de la tradition «melk» et «arch» en Algérie, in Guignard, D. et Guéno, V. (Dir.), Les acteurs des transformations foncières autour de la Méditerranée au XIX ${ }^{e}$ siècle, Paris: Karthala-IREMAM, p.49-93.

Gutiérrez, R. (2004) Internal Colonialism: An American Theory of Race, Du Bois Review: Social Science Research on Race, 1, 2, p. 281-295.

Hagan, J. (2010) Who are the Criminals? The Politics of Crime Policy from the Age of Roosevelt to the Age of Reagan, Princeton University Press.

Hayes, C. (2017) A Colony in a Nation, New York: W.W. Norton \& Company.

Hinton, E. (2016) From the War on Poverty to the War on Crime: The Making of Mass Incarceration in America, Cambridge: Harvard University Press. 
Hobsbawm, E. et Ranger, T. (Eds.) (1983) The Invention of Tradition, Cambridge University Press.

Hodge, J., Hödl, G. et Kopf, M. (Eds.) (2014) Developing Africa: Concepts and practices in Twentieth-Century Colonialism, Manchester University Press.

Houte, A.-D. et Luc, J.-N. (Dir.) (2016) Les gendarmeries dans le monde, de la Révolution française à nos jours, Paris: PUPS.

Jobard, F. (2006) Sociologie politique de la racaille, in Lagrange, H. et Oberti, M. (Dir.), Émeutes et protestations: une singularité française, Paris: Presses de Sciences Po, p. 59-80.

Jobard, F. et Lévy, R. (2009) Profiling Minorities. A study of Stop-and-Search Practices in Paris, Paris: Open Society Justice Initiative.

Jobard, F. et Nevanen, S. (2007) La couleur du jugement. Discriminations dans les décisions judiciaires en matière d'infractions à agents de la force publique (1965-2005), Revue française de sociologie, 48, 2, p. 243-272.

Kidambi, P. (2004) The Ultimate Masters of the City: Police, Public Order and the Poor in Colonial Bombay, c. 1893-1914, Crime, Histoire \& Sociétés/Crime, History \& Societies, 8,1, p. 27-47.

Kolsky, E. (2005) Codification and the Rule of Colonial Difference: Criminal Procedure in British India, Law and History Review, 23, 3, p. 631-683.

Kolsky, E. (2010) Colonial Justice in British India: White Violence and the Rule of Law, Cambridge University Press.

Lefeuvre, D. (1997) Chère Algérie. Comptes et mécomptes de la tutelle coloniale,1930-1962, Saint-Denis: Société française d'histoire d'outre-mer.

Locher-Scholten, E. (2002) State Violence and the Police in Colonial Indonesia, in Colombjin, F. et Lindbblad, J. (Eds.), Roots of Violence in Indonesia. Contemporary Violence in Historical Perspective, Leiden: KITLV Press.

Major A. (1999) State and Criminal Tribes in Colonial Punjab: Surveillance, Control and Reclamation of the "Dangerous Classes", Modern Asian Studies, 33, 3, p. 657-688.

Malye, F. et Stora, B. (2010) François Mitterrand et la guerre d'Algérie, Paris: Calmann-Lévy.

Mann, G. (2009) What was the Indigénat? The "Empire of Law" in French West Africa, The Journal of African History, 50, 3, p. 331-353.

Mann, M. (1984) The Autonomous Power of the State: its Origins, Mechanisms and Results, European Journal of Sociology, 25, 2, p. 185-213.

Mbembe, A. (2001) On the Postcolony, Berkeley, University of California Press.

Mohammed, M. et Mucchielli, L. (Dir.) (2007) Les bandes de jeunes. Des «blousons noirs» à nos jours, Paris: La Découverte.

Mucchielli, L. (1997) Sociologie versus anthropologie raciale. L'engagement des sociologues durkheimiens dans le contexte «fin de siècle» (1885-1914), Gradhiva. Revue d'histoire et d'archives de l'anthropologie, 21, p.77-95.

Morris, A. (2015) The Scholar Denied: W.E.B. Du Bois and the Birth of Modern Sociology, Oakland: University of California Press.

O'Reilly, C. (Ed.) (2017) Colonial Policing and the Transnational Legacy: the Global Dynamics of Policing across the Lusophone Community, London: Ashgate Publishing.

Osborne, M. (2014) Controlling Development: "Martial Race” and Empire in Kenya, 1945-59, The Journal of Imperial and Commonwealth History, 42, 3, p. 1-22. 
Petrow, S. (2000) Policing in a Penal Colony: Governor Arthur's Police System in Van Diemen's Land, 1826-1836, Law and History Review, 18, 2, p. 351-396.

Petrow, S. (2011) Bagnards ou Hommes libres : la police en Terre de Van Diemen, 18471858, in Denis, V., Denys, C. (Dir.), Polices d'Empires. XVIII ${ }^{e}$-XIX ${ }^{e}$ siècles, Rennes: PUR, p. 29-46.

Piazza, P. (2004) Histoire de la carte nationale d'identité, Paris: Odile Jacob.

Quijano, A. (2000) Coloniality of Power, Eurocentrism, and Latin America, Nepantla: Views from South, 1, 3, p. 533-580.

Renucci, F. (2011) La doctrine coloniale en République. L'exemple de deux jurisconsultes algériens : Marcel Morand et Emile Larcher, in Halpérin, J.-L. et Stora-Lamarre, A. (Dir.), La République et son droit (1870-1930), Besançon: Presses Universitaires de Franche Comté, p.461-478.

Riccio V., Ruediger M., Ross S. et al. (2013) Community policing in the Favelas of Rio de Janeiro, Police Practice and Research, 14, 4, p.308-318.

Roberts, R. (2005) Litigants and households: African disputes and colonial courts in the French Soudan, 1895-1912, Portsmouth, Heinemann.

Roberts, R. (2013) Law, Crime and Punishment in Colonial Africa, in Parker, J. et Reid, R. (Eds.), The Oxford Handbook of Modern African History, Oxford University Press, p. 171-188.

Rodet, M. (2009) Le délit d'abandon de domicile conjugal ou l'invasion du pénal colonial dans les jugements des «tribunaux indigènes » au Soudan français, 1900-1947, French Colonial History, 10, p. 151-169.

Sanchez, J.-L. (2013) À perpétuité : relégués au bagne de Guyane, Paris, Vendémiaire.

Särkkä, T. (2015) Imperialists without an empire? Finnish Settlers in Late Nineteenth- and early twentieth-century Rhodesia, Journal of Migration History, 1, 1, p.75-99.

Sassen, S. (2015) Old Borders and New Bordering Capabilities: Cities as Frontier Zones, Scienza \& Politica, 53, p. 295-306.

Sherman, T. (2009) Tensions of Colonial Punishment: Perspectives on Recent Developments in the Study of Coercitive Networks in Asia, Africa and the Carribean, History Compass, 7, 3, p. 659-677.

Singaravélou, P. (2011) Professer l'Empire. Les «sciences coloniales» en France sous la III République, Paris: Publications de la Sorbonne.

Skogan, W. (2003) Community Policing: Can It Work?, Belmont: Cengage Learning.

Steinmetz, G. (2008) The Colonial State as a Social Field: Ethnographic Capital and Native Policy in the German Overseas Empire before 1914, American Sociological Review, 73, 4, p. 589-612.

Stoler, A. (2009) Along the Archival Grain: Epistemic Anxieties and Colonial Common Sense, Princeton University Press.

Stoler, A. (2016) Duress. Imperial Durabilities in Our Times, Duke University Press.

Sundquist, E. (Ed.) (1996) The Oxford W.E.B. Du Bois reader, Oxford University Press.

Thénault, S. (2001) Une drôle de justice. Les magistrats dans la guerre d'Algérie, Paris: La Découverte.

Thénault, S. (2012) Violence ordinaire dans l'Algérie coloniale. Camps, internements, assignations à résidence, Paris: Odile Jacob. 
Thomas, M. (2008) Empire of Intelligence. Security Services and Colonial Disorder after 1914, Berkeley: University of California Press.

Thomas, M. (2012) Violence and Colonial Order: Police, Workers and Protest in the European Colonial Empires, 1918-1940, Cambridge University Press.

Tonry, M. (2007) Determinants of Penal Policies, Crime and Justice, 36, 1, p. 1-48.

Tonry, M. (2009) Emerging Explanations of American Punishment Policies, Punishment \& Society, 11, p.374-399.

Tonry, M. (2011) Punishing Race: A Continuing American Dilemma, Oxford: Oxford University Press.

Wacquant, L. (2009) Punishing the Poor: The Neoliberal Government of Social Insecurity, Durham: Duke University Press.

Wartelle, J.-C. (2004) La Société d'Anthropologie de Paris de 1859 à 1920, Revue d'Histoire des Sciences Humaines, 10, p. 125-171.

Weaver, J. (2003) The Great Land Rush and the Making of the Modern World, 1650-1900, Montreal: McGill University Press.

Wiener, M. (2009) An Empire on Trial: Race, Murder, and Justice under British Rule, 18701935, Cambridge University Press.

Wolfe P. (1999) Settler Colonialism and the Transformation of Anthropology, London: A\&C Black.

Zauberman, R., Robert, P., Névanen, S. et Bon, D. (2013) Victimation et insécurité en Île-deFrance. Une analyse géo-sociale, Revue française de sociologie, 54, 1, p. 111-153. 